\title{
Efficacy and Safety of Apatinib Treatment for Patients with Advanced Intrahepatic Cholangiocarcinoma
}

This article was published in the following Dove Press journal: Cancer Management and Research

\author{
Guohe Lin',* \\ Bicheng Wang (iD) ${ }^{2, *}$ \\ Xiuwei Wu' \\ Tong Sun' \\ Lili Chen' \\ Canliang Lu (iD) ${ }^{3}$ \\ Nianfei Wang' \\ 'Department of Oncology, The Second \\ Affiliated Hospital of Anhui Medical \\ University, Hefei, Anhui 23060I, People's \\ Republic of China; ${ }^{2}$ Cancer Center, \\ Union Hospital, Tongji Medical College, \\ Huazhong University of Science and \\ Technology, Wuhan 430022, People's \\ Republic of China; ${ }^{3}$ Department of \\ Hepatopancreatobiliary Surgery, \\ Department of General Surgery, The \\ First Affiliated Hospital of Anhui Medical \\ University, Hefei, Anhui 230022, People's \\ Republic of China
}

*These authors contributed equally to this work

Correspondence: Nianfei Wang Department of Oncology, The Second Affiliated Hospital of Anhui Medical University, Furong Road No. 678, Hefei, Anhui 23060I, People's Republic of China Email wangnianfei@foxmail.com

Canliang Lu

Department of Hepatopancreatobiliary Surgery, Department of General Surgery, The First Affiliated Hospital of Anhui Medical University, Jixi Avenue No. 218, Hefei, Anhui 230022, People's Republic of China

Email lucanliang@163.com
Purpose: Effective treatment options for intrahepatic cholangiocarcinoma (ICC) are limited. This study was intended to explore the efficacy and safety of apatinib in advanced ICC with lymph node metastasis or distant metastasis.

Patients and Methods: The efficacy and toxicity of apatinib were evaluated in patients with ICC between November 2017 and March 2020 at the Second Affiliated Hospital of Anhui Medical University. Survival analysis was estimated using Kaplan-Meier method.

Results: Ten patients with advanced ICC were enrolled. The median progression-free survival (PFS) was 3.0 months (95\% CI: 1.450-4.550). No patient achieved a complete response (CR). One patient gained partial response (PR), and 6 patients had stable disease (SD). The objective response rate (ORR) was $10 \%$, and the disease control rate (DCR) was $70 \%$. The common treatment-related adverse events were hypertension $(20 \%)$, proteinuria $(30 \%)$, hand and foot syndrome $(10 \%)$ or emesis $(10 \%)$. No grade $3 / 4$ toxicities occurred. Toxicities were mild and tolerable.

Conclusion: Apatinib is potentially an effective treatment option with tolerable toxicities for patients with advanced ICC.

Keywords: intrahepatic cholangiocarcinoma, apatinib, efficacy, toxicity, progression-free survival

\section{Introduction}

Intrahepatic cholangiocarcinoma (ICC) is a relatively rare tumor that arises from the intrahepatic duct. ${ }^{1,2}$ Surgery is the only radical method, but many patients fail to meet the requirement for surgery by the time they are diagnosed with the disease. Combining gemcitabine with cisplatin has been approved as the standard treatment for advanced ICC, but the efficacy remains limited. ${ }^{3}$ Therefore, new methods of treatment with improved efficacy are urgently needed to boost the lives of patients with ICC.

Because of the lack of effective driver genes, targeted therapies play a limited role in ICC. Multiple new drugs such as sorafenib, dabrafenib, and trametinib have failed to provide any satisfactory effect. ${ }^{4,5}$ Recently, some studies have shown that anti-angiogenic therapies are effective in treating some cancers. ${ }^{6}$ However, the effect of anti-angiogenic therapies on ICC is unclear.

Apatinib is an effective anti-angiogenic small molecule that not only improves the quality of life of tumor patients but also prolongs survival time. ${ }^{7,8}$ Recently, a case report demonstrated the efficacy of apatinib in the treatment of a 23-year-old female ICC patient after failed chemotherapy. ${ }^{9}$ However, for affirmation, further 
investigations with more ICC patients are needed to ascertain the efficacy of apatinib. Our study, therefore, was intended to explore the efficacy and safety of apatinib in the treatment of advanced ICC patients.

\section{Patients and Methods}

\section{Patients}

Ten patients with unresectable or relapsed ICC treated with apatinib were collected in Department of Oncology of the Second Affiliated Hospital of Anhui Medical University between November 2017 and March 2020. Eight of ten patients were pathologically confirmed as having ICC. The other 2 patients were clinically diagnosed as having ICC. All 10 patients had advanced or recurrent stage IV ICC according to the tumor, nodes, and metastases (TNM) classification. Nine patients received apatinib treatment after the failure of chemotherapy. Prior treatments before apatinib treatment included surgery, radiotherapy or chemotherapy. One patient refused to receive chemotherapy. Inclusion criteria consisted of the following: performance status (PS) 0 to 2; at least one measurable lesion without radiotherapy; no additional antitumor treatments administered at the same time as apatinib treatment; normal heart, liver and kidney functions. The main exclusion criteria were: uncontrolled hypertension; bleeding tendencies; a history of other tumors. Ethical approval (NO. YX2020-059(F1)) was obtained by the Ethical Committee of the Second Affiliated Hospital of Anhui Medical University in accordance with the Declaration of Helsinki. A written informed consent was provided by every patient.

\section{Treatment Methods}

Two hundred and fifty or five hundred milligram of apatinib was administered daily depending on the age, general status, and tolerance level of the patients. Responding or stable ICC patients maintained treatment until tumor progression or intolerable adverse events. The patients were followed-up until death or end of the research period.

\section{Response and Toxicity Assessments}

A treatment cycle lasted 28 days. Tumor response was evaluated after 2 treatment cycles or early when significant progression was judged to have occurred. A computed tomography (CT) scan was used to estimate the size of measurable lesions. Tumor response was assessed by the Response Evaluation Criteria in Solid Tumors (RECIST) guideline (version 1.1). Toxicities were assessed by the Common Terminology Criteria for Adverse Events (CTCAE) (version 5.0). Efficacy assessment encompassed complete response (CR), partial response (PR), stable disease (SD) or progressive disease (PD). The objective response rate (ORR) consisted of the percentages of $\mathrm{CR}$ and PR. The disease control rate (DCR) consisted of the percentages of CR, PR and SD.

\section{Data Analysis}

Statistical analyses were performed using SPSS 16.0. Median PFS was calculated using the Kaplan-Meier method.

\section{Results}

\section{Patient Characteristics}

Ten patients with advanced ICC were collected for this retrospective study. Eight of the ten patients were pathologically diagnosed as having ICC. The other two patients were clinically diagnosed with ICC. The characteristics of the 10 patients are illustrated in Table 1. Five patients $(50 \%)$ were male, and other 5 ones $(50 \%)$ were female. The daily dosage of apatinib was $250 \mathrm{mg}$ or $500 \mathrm{mg}$,

Table I Clinical Characteristics of the Study Population

\begin{tabular}{|l|l|}
\hline Variables & Numbers \\
\hline $\begin{array}{l}\text { Gender (\%) } \\
\text { Male } \\
\text { Female }\end{array}$ & $5(50)$ \\
\hline $\begin{array}{l}\text { Age, years (\%) } \\
\leq 50 \\
\geq 50\end{array}$ & $5(50)$ \\
\hline $\begin{array}{l}\text { ECOG PS (\%) } \\
0-I\end{array}$ & $2(50)$ \\
2 & $8(50)$ \\
\hline Clinical stage (\%) & \\
IVA & $7(50)$ \\
IVB & $3(50)$ \\
\hline Pathologic types (\%) & \\
Adenocarcinoma & $I(50)$ \\
None & $9(50)$ \\
\hline Treatment line (\%) & \\
First line & $8(50)$ \\
Second line & $2(50)$ \\
\hline Further line & \\
\hline
\end{tabular}




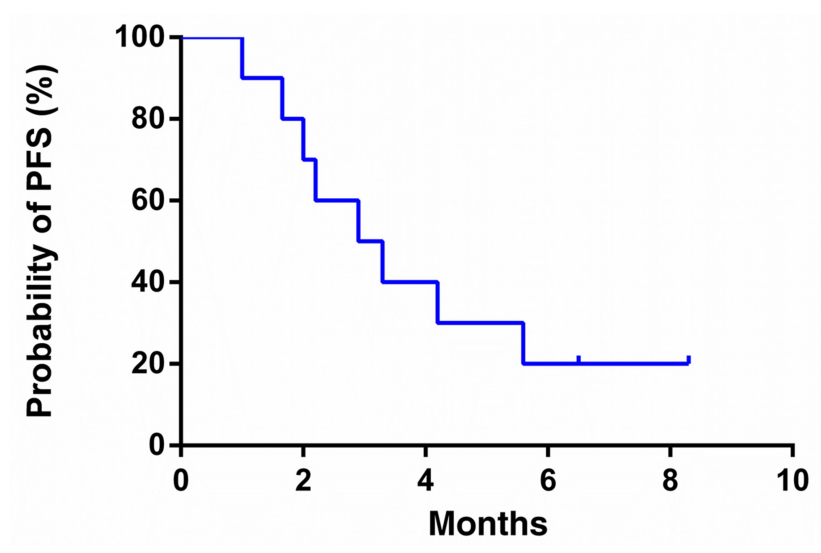

Figure I A progression-free survival curve for advanced ICC patients who received apatinib treatment.

depending on the age, general status, and tolerance level of the patients.

\section{The Efficacy of Apatinib Treatment}

The efficacy of apatinib was evaluated in 10 patients. No patient attained CR. One patient achieved PR, and 6 patients had SD. ORR was $10 \%$, and DCR was $70 \%$. The median PFS was 3.0 months (95\% CI: 1.450-4.550; Figure 1).

\section{Adverse Events}

Every patient was assessed for adverse events, which are summarized in Table 2. Toxicities were mild and tolerable. The treatment was not interrupted. No drug-related deaths occurred. Two patients suffered from hypertension. One patient experienced hand and foot syndrome. Three patients developed proteinuria. Two patients had symptoms of fatigue. One patient experienced emesis. Liver dysfunction was not observed. No grade 3/4 toxicities occurred.

\section{Discussion}

ICC is insensitive to chemotherapy or targeted therapy. Generally, it is hard to find an effective treatment method for patients with advanced diseases. However, recent

Table 2 The Toxicities of Apatinib Treatment

\begin{tabular}{|l|l|l|l|l|}
\hline \multirow{2}{*}{ Adverse Events } & \multicolumn{2}{|l|}{ Grades } & \multirow{2}{*}{$\begin{array}{c}\text { Total } \\
\text { n (\%) }\end{array}$} \\
\cline { 2 - 4 } & $\mathbf{I}$ & $\mathbf{2}$ & $\mathbf{3 - 4}$ & \\
\hline Fatigue & 2 & 0 & 0 & $2(20)$ \\
Hypertension & 0 & 2 & 0 & $2(20)$ \\
Hand-foot skin reaction & $\mathrm{I}$ & 0 & 0 & $\mathrm{I}(10)$ \\
Proteinuria & $\mathrm{I}$ & 2 & 0 & $3(30)$ \\
Emesis & 0 & $\mathrm{I}$ & 0 & $\mathrm{I}(10)$ \\
\hline
\end{tabular}

developments suggest that there is hope. ${ }^{9}$ Antiangiogenic inhibitors, including apatinib, have been shown to inhibit tumor development in some cancers. ${ }^{8}$

Apatinib is one oral small-molecule angiogenesis inhibitor that could inhibit tumor growth by targeting VEGFR-2. The disease control rate of apatinib was $67.4 \%$ in advanced non-small-cell lung cancer, and the median survival time was 5.8 months. ${ }^{10}$ Apatinib has also prolonged the median overall survival (OS) of advanced gastric cancer patients who received it, compared to placebo after chemotherapy failure. ${ }^{11}$ Likewise, a case report showed that apatinib was effective in treating ICC, after a 23-year-old advanced ICC female patient treated with apatinib, following chemotherapy failure, had a progression-free survival period of 6 months. ${ }^{9}$ These findings, particularly the one with the ICC patient, suggest that apatinib treatment could be a useful and promising method for ICC treatment. However, in the case of the research with the one patient with ICC, one patient is not enough to make a major claim. Therefore, the efficacy of apatinib in multiple ICC cases was of necessity.

Our study demonstrated that ORR of patients given apatinib was $10 \%$, and the median PFS was 3.0 months, suggesting that apatinib could prolong PFS. Nevertheless, our findings also showed that the effect of the drug was limited. It is, hence, unquestionable that combining apatinib with other treatments, such as immunotherapy, could provide a major boost and needs to be investigated. ${ }^{12}$ The limitation in this study is that the number of ICC patients is relatively less.

It is important to select an appropriate dose of apatinib for the treatment of patients. A daily dose of $850 \mathrm{mg}$ of apatinib is recommended. It has, however, been shown that a daily dose of $500 \mathrm{mg}$ of apatinib had similar efficacy to that of a high dose, and its grades 3 or 4 toxicities decreased. ${ }^{13}$ In another study, a daily dose of $250 \mathrm{mg}$ given to patients with non-small cell lung cancer produced a median PFS of 4 months. The disease control rate in that research was $61.7 \%$, there were no grade $3 / 4$ adverse effects, and 2 patients achieved PR. ${ }^{14}$ Our study showed that a daily dose of $500 \mathrm{mg}$ or $250 \mathrm{mg}$ of apatinib was effective in treating patients with ICC. As a result, the dose of apatinib can be adjusted according to patient conditions. If toxicities are intolerable, the dose of apatinib should be decreased. For patients with poor tolerance, a low daily dose of $250 \mathrm{mg}$ is recommended to start with, and then gradually increase to a daily dose of $500 \mathrm{mg}$.

Various side effects have been observed during apatinib treatment in different tumors. The common side effects of apatinib in hepatocellular carcinoma are fatigue, vomiting, hand-foot syndrome, hypertension, and hematologic toxicities. 
The most common grade 3 nonhematologic or hematologic toxicities are hypertension $(48.39 \%)$ or thrombocytopenia (6.45\%), respectively. ${ }^{15}$ For lung cancer, the main toxicities of apatinib have been identified as hypertension, hand-foot syndrome, and proteinuria. ${ }^{16}$ Here, we revealed that the side effects, including hand and foot syndrome or hypertension, were mild, meaning that the toxicities were tolerable. Compared with other angiogenesis inhibitors, the side effects of apatinib are similar or less. ${ }^{13,17,18}$ Therefore, apatinib could be considered a safe and promising alternative therapy for patients with cancer after multiline treatments.

\section{Conclusion}

This study showed that apatinib was an effective treatment option for patients with advanced ICC. Also, toxicities were mild and tolerable. However, further investigations, such as the suitable dosage of apatinib or combining apatinib with immunotherapy, are required for more affirmative inferences.

\section{Acknowledgments}

This research was supported by the Provincial Natural Science Research Project of Anhui Colleges (KJ2017A200), the Hubei Provincial Natural Science Foundation (Grant number: 2020CFB397 to Bi-Cheng Wang), and the Independent Innovation Foundation of WuHan Union Hospiatal (Grant number: 2019-109 to Bi-Cheng Wang).

\section{Disclosure}

The authors declare that they have no conflicts of interest.

\section{References}

1. Wei M, Lu L, Lin P, et al. Multiple cellular origins and molecular evolution of intrahepatic cholangiocarcinoma. Cancer Lett. 2016;379 (2):253-261. doi:10.1016/j.canlet.2016.02.038

2. Simone $\mathrm{V}$, Brunetti $\mathrm{O}$, Lupo L, et al. Targeting angiogenesis in biliary tract cancers: an open option. Int J Mol Sci. 2017;18(2):418. doi:10.3390/ijms 18020418

3. Weigt J, Malfertheiner P. Cisplatin plus gemcitabine versus gemcitabine for biliary tract cancer. Expert Rev Gastroenterol Hepatol. 2010;4 (4):395-397. doi:10.1586/egh.10.45

4. Pan TT, Wang W, Jia WD, et al. A single-center experience of sorafenib monotherapy in patients with advanced intrahepatic cholangiocarcinoma. Oncol Lett. 2017;13(5):2957-2964. doi:10.3892/ol.2017.5847
5. Lavingia V, Fakih M. Impressive response to dual BRAF and MEK inhibition in patients with BRAF mutant intrahepatic cholangiocarcinoma-2 case reports and a brief review. J Gastrointest Oncol. 2016;7(6):E98-E102. doi:10.21037/jgo.2016.09.13

6. Elkrief A, Alcindor T. Molecular targets and novel therapeutic avenues in soft-tissue sarcoma. Curr Oncol. 2020;27:34-40.

7. Chen Y, Ma G, Su C, et al. Apatinib reverses alectinib resistance by targeting vascular endothelial growth factor receptor 2 and attenuating the oncogenic signaling pathway in echinoderm microtubule-associated protein-like 4-anaplastic lymphoma kinase fusion gene-positive lung cancer cell lines. Anticancer Drugs. 2018;29(10):935-943.

8. Scott L. Apatinib: a review in advanced gastric cancer and other advanced cancers. Drugs. 2018;78(7):747-758. doi:10.1007/s40265018-0903-9

9. Wang LY, Gong S, Gao LP, et al. Apatinib for treating advanced intrahepatic cholangiocarcinoma after failed chemotherapy: a case report and literature review. Medicine. 2018;97(49):e13372. doi:10.1097/MD.0000000000013372

10. Leng J, Li DR, Huang LM, et al. Apatinib is effective as third-line and more treatment of advanced metastatic non-small-cell lung cancer: a retrospective analysis in a real-world setting. Medicine. 2019;98(36):e16967. doi:10.1097/MD.0000000000016967

11. Li J, Qin S, Xu J, et al. Randomized, Double-Blind, Placebo-Controlled Phase III trial of apatinib in patients with chemotherapy-refractory advanced or metastatic adenocarcinoma of the stomach or gastroesophageal junction. J Clin Oncol. 2016;34(13):1448-1454. doi:10.1200/ JCO.2015.63.5995

12. Liang L, Wen $\mathrm{Y}, \mathrm{Hu} \mathrm{R}$, et al. Safety and efficacy of PD-1 blockade-activated multiple antigen-specific cellular therapy alone or in combination with apatinib in patients with advanced solid tumors: a pooled analysis of two prospective trials. Cancer Immunol Immunother. 2019;68(9):1467-1477. doi:10.1007/s00262-019-02375-Z

13. Hu X, Zhang J, Xu B, et al. Multicenter Phase II study of apatinib, a novel VEGFR inhibitor in heavily pretreated patients with metastatic triple-negative breast cancer. Int $J$ Cancer. 2014;135 (8):1961-1969. doi:10.1002/ijc.28829

14. Liu Z, Ou W, Li N, et al. Apatinib monotherapy for advanced non-small cell lung cancer after the failure of chemotherapy or other targeted therapy. Thorac Cancer. 2018;9(10):1285-1290. doi:10.1111/1759-7714.12836

15. Yu WC, Zhang KZ, Chen SG, et al. Efficacy and safety of apatinib in patients with intermediate/advanced hepatocellular carcinoma: a prospective observation study. Medicine. 2018;97(3):e9704. doi:10. 1097/MD.0000000000009704

16. Fang SC, Zhang HT, Zhang YM, et al. Apatinib as post second-line therapy in EGFR wild-type and ALK-negative advanced lung adenocarcinoma. Onco Targets Ther. 2017;10:447-452. doi:10.2147/ OTT.S126613

17. Wu F, Zhang S, Xiong A, et al. A Phase II clinical trial of apatinib in pretreated advanced non-squamous non-small-cell lung cancer. Clin Lung Cancer. 2018;19(6):e831-e842. doi:10.1016/j.cllc.2018.06.002

18. Xiao B, Wang W, Zhang D. Risk of bleeding associated with antiangiogenic monoclonal antibodies bevacizumab and ramucirumab: a meta-analysis of 85 randomized controlled trials. Onco Targets Ther. 2018;11:5059-5074. doi:10.2147/OTT.S166151
Cancer Management and Research is an international, peer-reviewed open access journal focusing on cancer research and the optimal use of preventative and integrated treatment interventions to achieve improved outcomes, enhanced survival and quality of life for the cancer patient.
The manuscript management system is completely online and includes a very quick and fair peer-review system, which is all easy to use. Visit http://www.dovepress.com/testimonials.php to read real quotes from published authors. 